\title{
Dynamics of Primary and Secondary Infection in Take-All Epidemics
}

\author{
D. J. Bailey and C. A. Gilligan
}

Department of Plant Sciences, University of Cambridge, Downing Street, Cambridge, CB2 3EA, England, U.K. Accepted for publication 4 October 1998.

\begin{abstract}
Bailey, D. J., and Gilligan, C. A. 1999. Dynamics of primary and secondary infection in take-all epidemics. Phytopathology 89:84-91.

Using a combination of experimentation and mathematical modeling, the effects of initial (particulate) inoculum density on the dynamics of disease resulting from primary and secondary infection of wheat by the take-all fungus, Gaeumannomyces graminis var. tritici, were tested. A relatively high inoculum density generated a disease progress curve that rose monotonically toward an asymptote. Reducing the initial inoculum density resulted in a curve that initially was monotonic, rising to a plateau, but which increased sigmoidally to an asymptotic level of disease thereafter. Changes in the infectivity of particulate inoculum over time were examined in a separate experiment. Using a model that incorporated terms for primary and secondary infection, inoculum decay, and host growth, we showed that both disease progress curves were consistent

with consecutive phases dominated, respectively, by primary and secondary infection. We examined the spread of disease from a low particulate inoculum density on seminal and adventitious root systems separately. Although seminal roots were affected by consecutive phases of primary and secondary infection, adventitious roots were affected only by secondary infection. We showed that the characteristic features of disease progress in controlled experiments were consistent with field data from crops of winter wheat. We concluded that there is an initial phase of primary infection by G. graminis var. tritici on winter wheat as seminal roots grow through the soil and encounter inoculum, but the rate of primary infection slows progressively as inoculum decays. After the initial phase, there is an acceleration in the rate of secondary infection on both seminal and adventitious roots that is stimulated by the increase in the availability of infected tissue as a source of inoculum and the availability of susceptible tissue for infection.
\end{abstract}

The temporal spread of take-all disease on cereal roots, caused by Gaeumannomyces graminis (Sacc.) Arx \& D. Olivier var. tritici Walker, originates from dual sources of primary and secondary infection $(3,7,11,12)$. Primary infection is initiated from a declining reservoir of inoculum carried over from the previous cereal crop; secondary infection involves transmission by mycelial contact between infected and susceptible roots. Both forms of infection occur in a dynamically changing population of susceptible hosts as new roots are produced. Understanding the balance between primary and secondary infection is critical in optimizing deployment of new biological and chemical control methods that are becoming available for G. graminis var. tritici, for which the duration of control depends on the persistence of agents introduced on seed.

In a previous paper, Gilligan and Kleczkowski (14) used a combination of analytical and numerical methods to analyze the behavior of a generic model for primary and secondary infection with static, unlimited (exponential), and asymptotically limited host growth, with and without secondary infection, and with and without decay of initial inoculum. The model formula is

$$
\begin{aligned}
\text { Infected roots: } & \mathrm{d} I / \mathrm{d} t=\left(r_{p} P+r_{s} I\right)(N-I) \\
\text { Total roots: } & \mathrm{d} N / \mathrm{d} t=r_{n} f(N) \\
\text { Inoculum: } & \mathrm{d} P / \mathrm{d} t=-r_{d} P
\end{aligned}
$$

in which $r_{p}$ and $r_{s}$ are rates of primary and secondary infection, respectively, $r_{n}$ is the rate of root production, $r_{d}$ is the rate of inoculum decay, $f(N)$ is a general term for host growth that includes static, unlimited, and limited growth, $I$ and $N$ are the numbers of infected and total main (seminal and adventitious) root axes per unit area, respectively, $P$ is the density of inoculum per unit area for a fixed soil depth. The principal variables and param-

Corresponding author: D. J. Bailey; E-mail address: djb21@ cam.ac.uk

Publication no. P-1998-1124-02R

(c) 1999 The American Phytopathological Society eters used in the models are summarized in Table 1. The theoretical results showed that epidemics exhibit a range of behavior within single seasons, with disease progress curves extending from a simple monotonic increase with saturation of the host population, through temporary plateaus as the system switches from primary to secondary infection, to effective elimination of the pathogen when the host outgrows the fungal infection.

Analytical use of the models showed that the inoculum decay and relative growth of the host combine to affect the dynamics of infection. In particular, after an initial period of increase (driven by primary infection), the proportional amount of infection slowed down. Deceleration was caused by inoculum decay, which reduced the rate of primary infection before secondary infection began to build up. Whereas plants may outgrow epidemics, especially when the source of inoculum in soil decays (14), secondary infection can prevent this, resulting in saturation of roots with infection and disease. Although primary and secondary infection occur simultaneously during at least part of an epidemic, the shift from a phase of predominantly primary to secondary infection may lead to profound changes in epidemic rates, which may take the form of marked acceleration as the multiplicative effect of secondary infection occurs. A shift from primary to secondary infection can also, in certain circumstances, lead to a temporary or even persistent plateau in the period during which most primary infection is completed as the reservoir of soil inoculum decays to zero and a delay occurs before secondary infection accelerates.

Brassett and Gilligan (4) fitted simplified models related to equation 1 to field data for G. graminis var. tritici, but the results were equivocal because of the paucity of data. Similarly, Colbach et al. (7) recently fitted a model for primary and secondary infection, derived from the Brassett and Gilligan (3) model, to field data. The model did not include terms for host growth or inoculum decay. Moreover, assessment of disease did not commence until plants had reached stem elongation (growth stage 30 ), by which time the epidemic was well advanced. In the current paper, we use a combination of controlled environment experi- 
ments and model fitting to analyze the effects of primary and secondary infection on the dynamics of take-all of wheat. Specifically, the paper is designed to test whether there is (i) a detectable shift in phase from primary to secondary infection in the dynamics of take-all; (ii) a temporary plateau in disease progress between the two phases; and (iii) a change in epidemic rate as secondary infection increases with increasing density of adventitious roots.

The role of initial inoculum density in controlling the shape of the disease progress curve also is examined. The controlled environment experiments allow comparatively large numbers of sequential observations of infection and disease over time. The results are compared with data for large field experiments, and the consequences for epidemiology and control of take-all disease are discussed.

\section{MATERIALS AND METHODS}

Experimental and modeling strategies used to test main hypotheses. Disease progress curves were constructed for epidemics of G. graminis var. tritici in destructively sampled microcosms in controlled environments, for which the rates of inoculum decay and production of main root axes also were measured. The model for primary and secondary infection was fitted to disease progress curves initiated by two densities of initial inoculum to test the hypothesis of a temporary plateau in disease progress. The progress of disease was separated into curves for seminal and adventitious roots to test for a detectable switch in phase from primary to secondary infection in the two root systems. We used an independent method, based on the probability of contact between initial inoculum and roots, to estimate the maximum proportion of seminal roots that could be infected by primary infection; the switch to secondary infection in adventitious roots was analyzed by comparing the inoculum decay curve with the curve for disease progress on adventitious roots. The postulated mechanisms for disease progress derived from the controlled environment experiment produced characteristic curve shapes for the two root systems, which were compared with disease progress curves from large field experiments to test the applicability of the hypotheses to disease progress in the field.

Inoculum. The inoculum used in all experiments consisted of G. graminis var. tritici (isolate ML1) grown on foxtail millet (Setaria italica) grains, which provided small, uniform infection units. To produce inoculum, $12 \mathrm{~g}$ of sand, $12 \mathrm{~g}$ of millet, and $10 \mathrm{ml}$ of distilled water were added to a $100-\mathrm{ml}$ conical flask and autoclaved twice at $103.5 \mathrm{kPa}$ for $1 \mathrm{~h}$, with a 3-day interval between autoclavings. Flasks were incubated for 28 days at $19^{\circ} \mathrm{C}$ with six disks (4 mm diameter), cut from the margin of a G. graminis var. tritici colony grown on potato dextrose agar. Inoculum was stored in the dark at $4^{\circ} \mathrm{C}$.

Host species. Winter wheat cv. Riband was used in the experiments. Seeds were selected for uniformity of size, surface-sterilized for $5 \mathrm{~min}$ in $5 \%$ sodium hyperchlorite $(0.5 \%$ [wt/vol] available chlorine), and rinsed twice in sterile distilled water. Seeds were incubated for $48 \mathrm{~h}$ at $19^{\circ} \mathrm{C}$ and selected for uniformity of germination.

Soil. A clay soil (sandy clay loam, with a clay content of $23.8 \%$, organic matter content of $2.1 \%$, and a cation exchange capacity of $6.4 \mathrm{meq} / 100 \mathrm{~g}$ ) was obtained from the Cambridge University Field Station (Cambridge) from a site known to have been free of grass species for a minimum of 5 years. Soil was sieved through a $10-\mathrm{mm}$ screen, air-dried for 14 days, and sieved further through a 2-mm screen to remove stones and large pieces of debris. Soil was baited to certify that it was free of detectable G. graminis var. tritici inoculum and mixed with sand at a ratio of two parts soil to one part sand to aid drainage, aeration, and removal of soil prior to assessment. The soil-sand mixture (hereafter referred to as soil) had a field capacity of $21 \%$, corresponding to a water potential of $-0.015 \mathrm{MPa}$ and $\mathrm{H}_{2} \mathrm{O} \mathrm{pH}$ of 7.0.
Disease progress curves. Two batches of inoculum were prepared with 15 (low inoculum density) and 240 (high inoculum density) propagules (infested millet seeds) per 1,245 $\mathrm{g}$ of soil. Pots (160-mm deep, 150-mm internal diameter) were filled from the bottom up with $1,100 \mathrm{~g}$ of soil (50-mm deep), 1,100 $\mathrm{g}$ of soil (50-mm deep) with infested millet seed, and $880 \mathrm{~g}$ of soil (40-mm deep). Five pregerminated wheat seeds were planted in the center of the pot, covered with $220 \mathrm{~g}$ (10-mm deep) of soil, and topped with $250 \mathrm{~g}$ of sand (10-mm deep) to prevent excessive moisture evaporation. Pot size, host density, and planting position were selected to facilitate unrestricted root growth in the main region of disease spread (i.e., within and above the inoculum layer). Pots were allowed to stand in water until saturated, after which they were transferred to a growth cabinet.

The experiment included 20 pots each at the two inoculum densities that were fully randomized between two growth cabinets. Soil moisture was maintained just below field capacity $(-0.015 \mathrm{MPa})$ by monitoring eight pots daily, four from each treatment, using a soil moisture probe (Type 2900F, Soil Moisture Equipment Corp., Santa Barbara, CA) and adding water as required. Pots were incubated at $15^{\circ} \mathrm{C}$ with a regime of $16 \mathrm{~h}$ of light and $8 \mathrm{~h}$ of dark. Plants were assessed over a 12 -week period by washing soil from roots and carefully separating them. Roots of each plant were examined under a binocular microscope. The numbers of diseased and healthy main root axes on both seminal and adventitious root systems were recorded; disease was defined as stelar discoloration. The numbers provided estimates for changes in total root density over time, $N(t)$.

Two additional pots were included to estimate the number and average length of seminal roots passing through the inoculum layer. The data were used to estimate the maximum level of primary infection on seminal roots.

Inoculum decay. Estimates of changes in the density of infective particulate inoculum over time, $P(t)$, were obtained in a separate experiment. The experiment was performed in two parts. In the first part, inoculum was placed in soil packs (described below) in the presence of the host plant and allowed to decay over time. In the second part, units of inoculum were removed at different time intervals and assayed for infectivity by placement against fresh roots of wheat plants also grown in soil packs.

Soil packs were prepared from 200-mm lengths of 70-mm-diameter, clear Layflat polyethylene tubing (Layflat Tubing, Isle of Wight,

TABLE 1. Principal variables, parameters, and functions used in the models

\begin{tabular}{|c|c|c|}
\hline Symbol & Description & Unit $^{\mathrm{a}}$ \\
\hline \multicolumn{3}{|l|}{ Variable } \\
\hline$I$ & Infected roots & Number $\mathrm{cm}^{-3}$ \\
\hline$N$ & Total roots & Number $\mathrm{cm}^{-3}$ \\
\hline$P$ & Propagules of inoculum & Number $\mathrm{cm}^{-3}$ \\
\hline$i$ & Proportion of infected roots & $\mathrm{cm}^{-3}$ \\
\hline$I_{p}$ & Seminal roots infected by primary infection & Number $\mathrm{cm}^{-3}$ \\
\hline \multicolumn{3}{|c|}{ Parameter } \\
\hline$r_{p}$ & Primary infection rate & Day $^{-1}$ \\
\hline$r_{s}$ & Secondary infection rate & Day $^{-1}$ \\
\hline$r_{n}$ & Root production rate & Day $^{-1}$ \\
\hline$r_{d}$ & Inoculum decay rate & Day $^{-1}$ \\
\hline$\phi$ & Probability of root infection by a single propagule & \\
\hline$\zeta$ & $\begin{array}{l}\text { Probability of a single propagule occurring in } \\
\text { pathozone }\end{array}$ & \\
\hline$\psi$ & $\begin{array}{l}\text { Probability of a single propagule from a random } \\
\text { location in pathozone causing infection }\end{array}$ & \\
\hline \multicolumn{3}{|l|}{ Function } \\
\hline$f(N)$ & Root growth response & Number $\mathrm{cm}^{-3}$ \\
\hline$G(t)$ & Relative growth rate of roots & Day $^{-1}$ \\
\hline
\end{tabular}

${ }^{a}$ Units are expressed as number of roots or propagules per unit volume, comprising, for pots, surface area multiplied by fixed depth of inoculum layer within pots and, for field, samples surface area multiplied by depth $(\approx 15 \mathrm{~cm})$ from which roots were removed. 
England, U.K.). The tubing was partially sealed at one end using staples and filled with $375 \mathrm{~g}$ of moist soil $(10 \mathrm{ml}$ of water per $125 \mathrm{~g}$ of soil). Packs were sealed at the top, allowing enough room for emergence of a coleoptile, and compressed to achieve enough rigidity to stand upright. Pregerminated seeds were introduced into the pack by making a small incision, inserting the seed, and resealing with clear, adhesive tape. Soil moisture was adjusted to near field capacity, and the packs were placed in the growth chamber. Ten propagules were introduced into each of ten packs at random locations.

On each of 10 sampling occasions $(0,4,6,9,10,12,16,21,24$ and 33 days), one propagule was removed from each pack. Propagules were tested for the ability to cause disease by placement against the root tips of 7-day-old wheat roots grown in additional soil packs. Soil packs were arranged in a fully randomized block design, and roots were assessed for stelar discoloration after 14 days. The proportions of successful infection were used to estimate changes in inoculum density over time, $P(t)$. Both parts of the experiment were performed under the conditions described above for production of disease progress data.
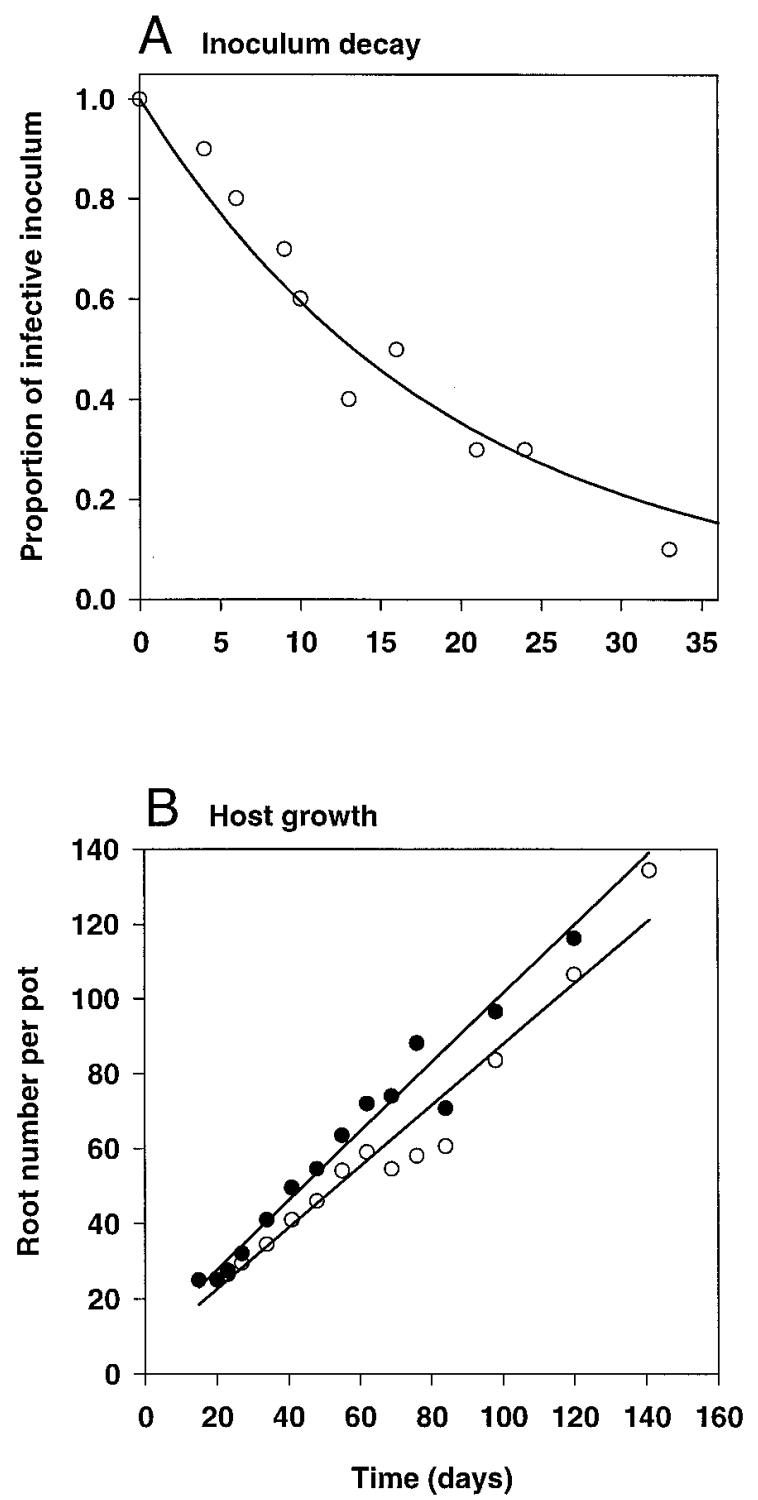

Fig. 1. Changes in A, proportion of infective particulate inoculum and $\mathbf{B}$, number of main root axes per pot over time. The fitted curve for inoculum decay is given by $p(t)=\exp (-0.052 t)$. Linear growth rates for density of main root axes are given by $N(t)=0.81 t$ and $N(t)=0.92 t$ for plants infected from low (open circles) and high (closed circles) densities of particulate inoculum, respectively.
Model fitting. Inoculum decay and host growth. A simple, exponential model, of the form $P(t)=P(0) \exp \left(-r_{d} t\right)$,was fitted to changes in inoculum density, $P(t)$, with age, $t$, of inoculum, under the assumption of a binomial error distribution using the maximum likelihood package (22). Data for changes in the total number of roots over time were described by a simple rectilinear model.

Disease progress. To fit differential equation models (equation 1) to disease progress data, it is convenient to introduce dimensionless variables to scale disease relative to changing host size: if $i=$ $I / N$ is the proportion of infected roots, then according to Gilligan and Kleczkowski (14)

$$
\mathrm{d} i / \mathrm{d} t=\left[r_{p} P+\rho_{s}(t) i\right](1-i)-G(t) i
$$

where

$$
\rho_{s}(t)=r_{s} N \text { and } G(t)=(1 / N)(\mathrm{d} N / \mathrm{d} t)=r_{n}[f(N) / N]
$$

Details of the derivation for model 2, which involves expansion of the quotient $(I / N)$ to obtain $\mathrm{d} i / \mathrm{d} t$, are given in Gilligan and Kleczkowski (14). Model 2, with and without allowance for host growth and inoculum decay, was fitted by least squares to disease progress data using the numerical curve-fitting software package Facsimile (AEA Technology, Harwell, U.K.) under the simplifying assumptions of normal errors with constant variance. Goodness-offit was assessed by comparing residual deviance (with and without allowance for host growth and inoculum decay) and residual error trends. The errors associated with individual observations also were examined, and the magnitudes of parameter estimates for primary and secondary infection were checked for biological plausibility.

Primary infection of seminal roots. The maximum proportion of seminal roots that could be infected by primary infection $\left(I_{p}\right)$ as they passed through the inoculum layer was estimated from simple probability models (9). Thus,

$$
I_{p}=1-(1-\phi)^{P(0)}
$$

where $P(0)$ is the initial density of inoculum and $\phi$ is the probability that a root is infected by a single propagule of inoculum. The method for calculation of $\phi$ is summarized briefly; a detailed account is given in Gilligan (10) for general cases of soilborne disease and in Bailey and Gilligan (2) for Rhizoctonia solani. The probability of infection is calculated from the product of $\phi=\zeta \psi$, where $\zeta$ is the probability that a propagule of inoculum lies within the pathozone surrounding a susceptible root and $\psi$ is the probability that a randomly located propagule within the pathozone can infect the host. The probability of occurrence within the pathozone is given by the ratio of the volume of the pathozone of radius $R$ surrounding a root of radius $h$ and length $l$ passing through the inoculum layer to the volume $(V)$ of the layer in which inoculum is located:

$$
\zeta=\left\{\pi /\left[(R+h)^{2}-h^{2}\right]\right\} / V
$$

$\psi$ is obtained as

$$
\psi=\int_{h}^{R_{0}} f(r) \cdot g(r) \mathrm{d} r
$$

where $f(r)=2(r+h) /\left[(R+h)^{2}-h^{2}\right]$ is the probability density for the location of the propagule within the pathozone and $g(r)=$ $\gamma \exp \left(-\delta r^{2}\right)$ is the probability that the propagule can infect (15). The parameters $(R, \gamma$, and $\delta)$ for $\zeta$ and $\psi$ were estimated from the placement experiments described above; root radius $(h)$ and length ( $l$ ) were measured.

Field data. Results from controlled environment experiments were compared with disease progress curves from a large field plot experiment (CS212) conducted during 1988 at Rothamsted Experimental Station (England, U.K.). The experiment involved plots $(3 \times$ $5 \mathrm{~m}$ ) of first, second, fourth, fifth, and continuous (11 years) wheat crops assessed over a 2-year period. Plants selected from two replicate plots were assessed for total and diseased seminal and adventitious roots from mid-April to harvest. Details of sampling 
and agronomy are given in Werker et al. (28). To minimize the effects of temperature on the shape of the disease progress curve, the independent variable (time) was transformed to day degrees $\left(\times 10^{-3}\right)$ above $3^{\circ} \mathrm{C}$ at $100 \mathrm{~mm}$ below the soil surface (from 15 October). The baseline of $3^{\circ} \mathrm{C}$ represents the minimum temperature for wheat growth (5). The sample dates of 186, 201, 214, $221,228,235,242,249,256,263,270,277$, and 284 days from sowing correspond to $623,715,828,889,960,1,026,1,094,1,179$, $1,275,1,359,1,442,1,522$, and 1,615 day degrees above $3^{\circ} \mathrm{C}$.

\section{RESULTS}

Inoculum decay and host growth. Particulate inoculum for primary infection decayed exponentially during the course of the experiments (Fig. 1A), according to $p(t)=\exp (-0.052 t)$, where $p=$ $P / P(0)$ is the proportion of surviving inoculum. Extrapolation of the function shows that the proportion of primary inoculum capable of causing infection after 50 days was less than 0.05 .

The total density of roots increased approximately linearly (Fig. 1B): $\mathrm{d} n / \mathrm{d} t=r_{n}$ and $f(N)=1$ in equation 1 , giving $G(t)=r_{n} / N$, where $r_{n}=0.81$ and 0.92 for low and high inoculum density treatments, respectively.
Disease progress curves and model fitting. The proportion of diseased roots increased monotonically to 0.9 after 140 days for the high inoculum density treatment (Fig. 2A). In the low inoculum density treatment, the proportion of diseased roots rose monotonically for 50 days, reaching a temporary plateau of 0.2 , followed by a sigmoidal increase in disease that culminated in an asymptote when the proportion of diseased roots was 0.9 after 140 days.

The primary and secondary infection model given in equation 2 , with linear host growth and exponential inoculum decay, was successfully fitted to the data (Table 2; Fig. 2A through C) and showed evidence of a temporary flattening of the curve for the low inoculum density treatment. Failure to allow for host growth $\left(N=N_{\max }\right)$ together with inoculum decay $(P=P(0))$ substantially reduced the goodness-of-fit of the model (Fig. 2D through F; Table 2). Allowance for host growth or inoculum decay alone each failed to reproduce the initial plateau for infection of total roots over time (data not shown).

Disease progress on seminal and adventitious roots. Disease progress from a low density of particulate inoculum was examined on seminal and adventitious roots separately. We fitted equation 2, including a term for inoculum decay but assuming a fixed density of roots, to disease progress on seminal roots, which assumes that
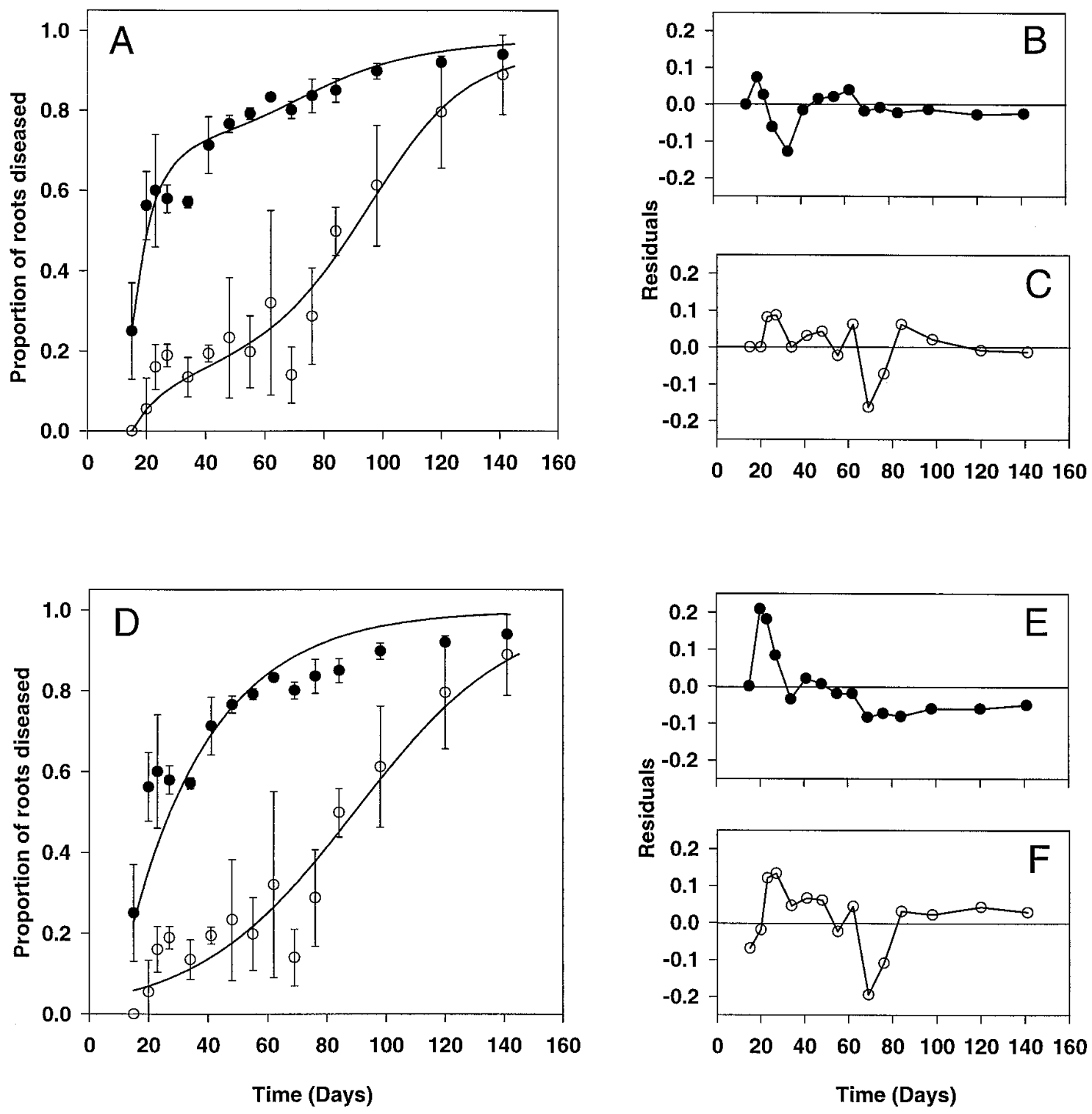

Fig. 2. Disease progress curves for low (open circles) and high (closed circles) densities of particulate inoculum in the controlled environment experiment. Data were fitted with a model (equation 2) including terms for primary and secondary infection $\mathbf{A}$, with or $\mathbf{D}$, without allowance for inoculum decay and growth (changes in numbers) of main root axes. B, C, E, and F, residual plots for goodness-of-fit of the model. 
most secondary infection on seminal roots originates from other seminal roots rather than adventitious roots, because of the partial spatial separation of roots. The pattern of disease on adventitious roots was fitted by a logistic function simply to summarize the char-
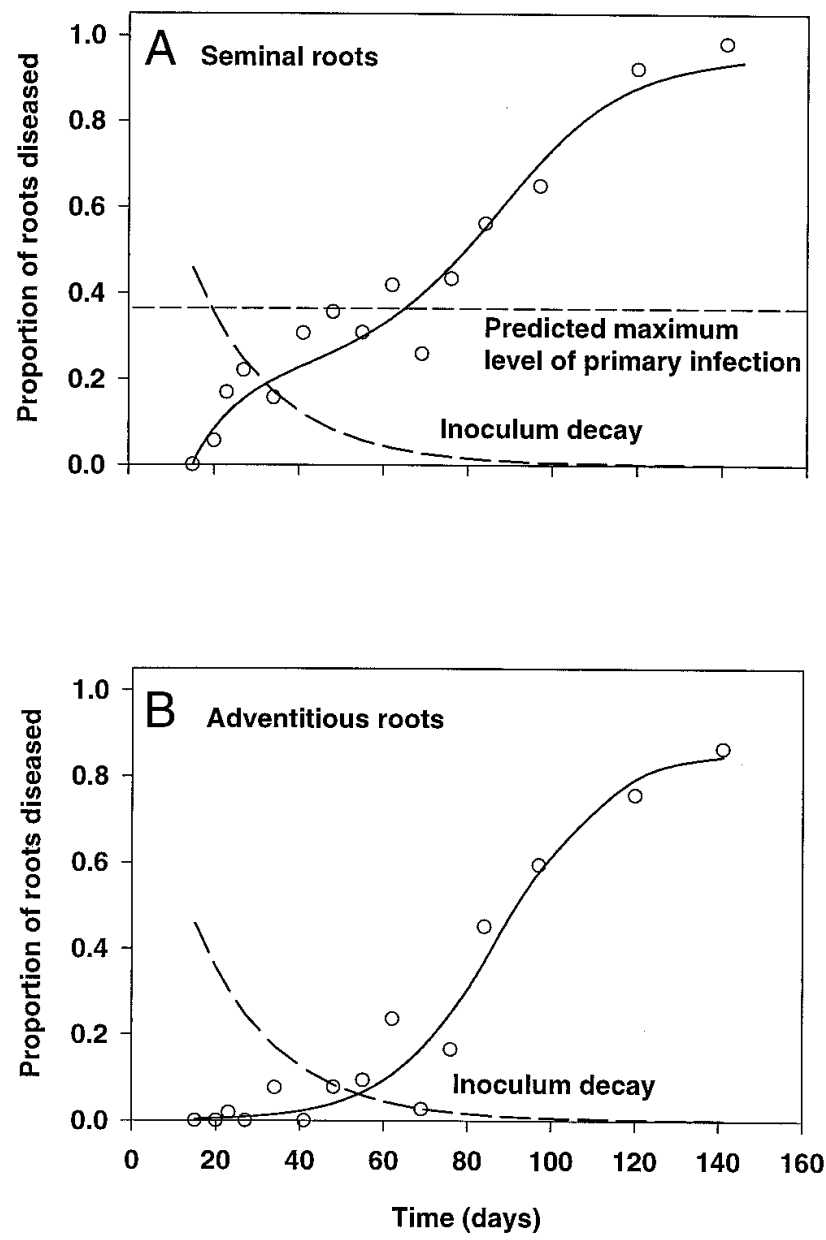

Fig. 3. Disease progress from a low density of particulate inoculum on A, seminal and $\mathbf{B}$, adventitious roots in the controlled environment experiment. Curves for decay of particulate inoculum and maximum level of primary infection (prediction based on equation 3) on seminal roots are shown. Progress of disease on seminal roots was fitted by the model for primary and secondary infection (equation 2), with a fixed density of seminal roots $(N=6$ per plant $)$ and exponential decay $\left(r_{d}=0.052\right)$ of particulate inoculum, giving estimates of $r_{p}=2.55 \times 10^{-3}$ and $r_{s}=5.72 \times 10^{-4}$, with a residual deviance of 7.75 on 12 df. The sigmoidal progress of disease on adventitious roots was summarized by a logistic equation: $i=0.86 /\{1+\exp [-0.076(t-87.7)]\}$. acteristic sigmoidal shape of disease progress. On the seminal root system, the shape of disease progress was similar to that for the entire root system. Disease increased monotonically, without a point of inflection, to an initial plateau when the ratio of diseased to nondiseased roots was $\approx 0.35$ (Fig. $3 \mathrm{~A}$ ), followed by an increase in the rate of infection that slowed as the proportion of diseased seminal roots approached 1.0. Disease progress on adventitious roots was sigmoidal, increasing at 30 days from no disease to a proportion of $\approx 0.8$ roots diseased after 140 days (Fig. $3 B$ ).

Primary infection of seminal roots. The asymptotic (longterm) probability that a seminal root passing through an inoculum layer of volume $V=883.6 \mathrm{~cm}^{3}$ was infected by primary infection from a low density $(P=13.25)$ of initial inoculum was estimated from placement experiments as $\phi=0.031$, where the mean root radius $(h)$ was $0.5 \mathrm{~mm}$, the mean root length $(l)$ was $80 \mathrm{~mm}$, and the width of the pathozone $(R)$ was $9 \mathrm{~mm}$. Parameters $\gamma$ and $\delta$, which describe changes in the probability of infecting a root with distance from the root surface $(\psi)$, were estimated from placement experiments as 1.0 and 0.032 respectively. The maximum proportion of seminal roots $\left(I_{p}\right)$ infectable by primary inoculum in the low inoculum density treatment was calculated as $I_{p}=0.342$ (Fig. 3A).

Field data. Examples of typical disease progress curves from field data for progress of take-all disease on total, seminal, and adventitious roots of first, second, fourth, fifth, and continuous (11 consecutive wheat crops) crops in a consecutive sequence of winter wheat crops are shown in Figure 4. Time has been transformed to day degrees above $3^{\circ} \mathrm{C}$ to minimize the effects of changing temperature during early spring. The model for primary and secondary infection was fitted to the data for seminal roots, with the rate of inoculum decay $\left(r_{d}\right)$ fixed (in the absence of additional information) across cropping frequency. The density of seminal roots was assumed to be constant over the course of the experiment. Trends in disease progress for adventitious roots were summarized by fitting an exponential model over the period of sampling.

The results of the field data were consistent with the decomposition results for the controlled environment experiment (comparison of Figs. 3 and 4). There were low levels of primary infection, which were primarily confined to seminal roots. By mid-April (equivalent to 600 day degrees above $3^{\circ} \mathrm{C}$ ), none of the adventitious roots were diseased, although there were between five and eight adventitious roots per plant, while the proportion of seminal roots, which showed symptoms of disease, was 0.05 to 0.15 . Previous work by Hornby (19) has shown that most initial inoculum of $G$. graminis var. tritici in soil from the Rothamsted site decayed by the onset of significant growth of adventitious roots during the spring. The remaining intensification of the epidemic, therefore, occurred by secondary infection on both seminal and adventitious

TABLE 2. Parameter estimates and residual deviances for fitting model for primary and secondary infection to data from the controlled environment experiment

\begin{tabular}{|c|c|c|c|c|c|}
\hline Model & $\begin{array}{c}\text { Inoculum } \\
\text { density }\end{array}$ & Parameter estimate & 95\% Confidence interval & $\begin{array}{l}\text { Residual } \\
\text { deviance }^{\mathrm{a}}\end{array}$ & df \\
\hline $\begin{array}{l}\text { Full model (including inoculum } \\
\text { decay and host growth) }\end{array}$ & Low & $\begin{array}{l}r_{p}=1.98 \times 10^{-3} \\
r_{s}=8.19 \times 10^{-4} \\
r_{d}=0.052 \\
r_{n}=0.81 \\
r_{p}=2.63 \times 10^{-3} \\
r_{s}=2.00 \times 10^{-3} \\
r_{d}=0.052 \\
r_{n}=0.92\end{array}$ & $\begin{array}{l}1.42 \times 10^{-3} \text { to } 2.76 \times 10^{-3} \\
6.86 \times 10^{-4} \text { to } 9.77 \times 10^{-4} \\
0.045-0.058 \\
0.761-0.859 \\
2.26 \times 10^{-3} \text { to } 3.00 \times 10^{-3} \\
1.55 \times 10^{-3} \text { to } 2.64 \times 10^{-3} \\
0.045-0.058 \\
0.878-0.962\end{array}$ & 7.32 & 12 \\
\hline $\begin{array}{l}\text { Reduced model (excluding inoculum } \\
\text { decay and host growth) }\end{array}$ & $\begin{array}{l}\text { Low } \\
\text { High }\end{array}$ & $\begin{array}{l}r_{p}=4.7 \times 10^{-4} \\
r_{s}=2.9 \times 10^{-2} \\
r_{p}=1.5 \times 10^{-4} \\
r_{s}=1.5 \times 10^{-6}\end{array}$ & $\begin{array}{c}2.27 \times 10^{-4} \text { to } 9.75 \times 10^{-4} \\
1.77 \times 10^{-2} \text { to } 4.91 \times 10^{-2} \\
1.46 \times 10^{-4} \text { to } 1.78 \times 10^{-2} \\
\ldots^{\text {b }}\end{array}$ & $\begin{array}{l}12.6 \\
13.0\end{array}$ & $\begin{array}{l}12 \\
14\end{array}$ \\
\hline
\end{tabular}

${ }^{a}$ Residual deviance $=$ residual sum of squares.

${ }^{\mathrm{b}}$ Parameter $r_{s}$ fixed, $r_{p}$ estimated. 
roots. The field data were variable, but we did note that the estimated plateau densities for primary infection of seminal roots increased with cropping frequency to the fourth consecutive wheat crop before leveling off and subsequently dropping with continuous cultivation (Fig. 4).

\section{DISCUSSION}

The dynamics of G. graminis var. tritici are complicated by the interactions of the host, disease, and inoculum dynamics, each of which may be affected by environmentally driven variables, such

\section{A Seminal roots}
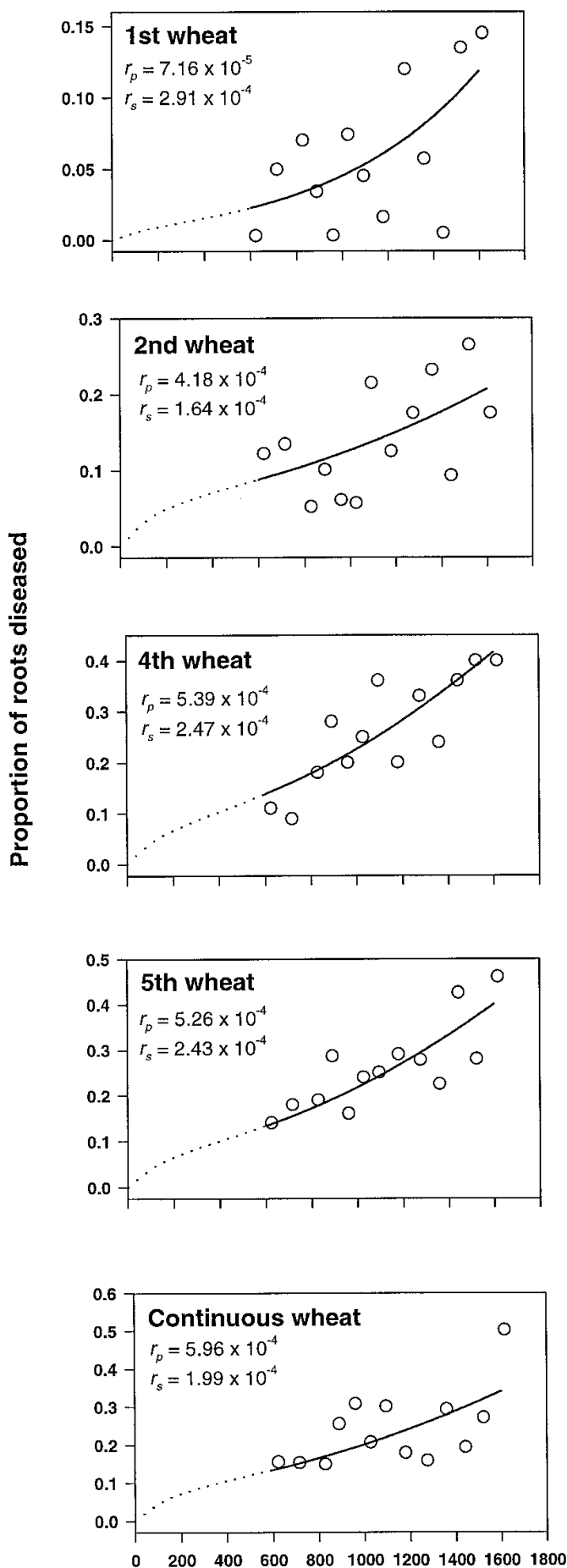

\section{B Adventitious roots}
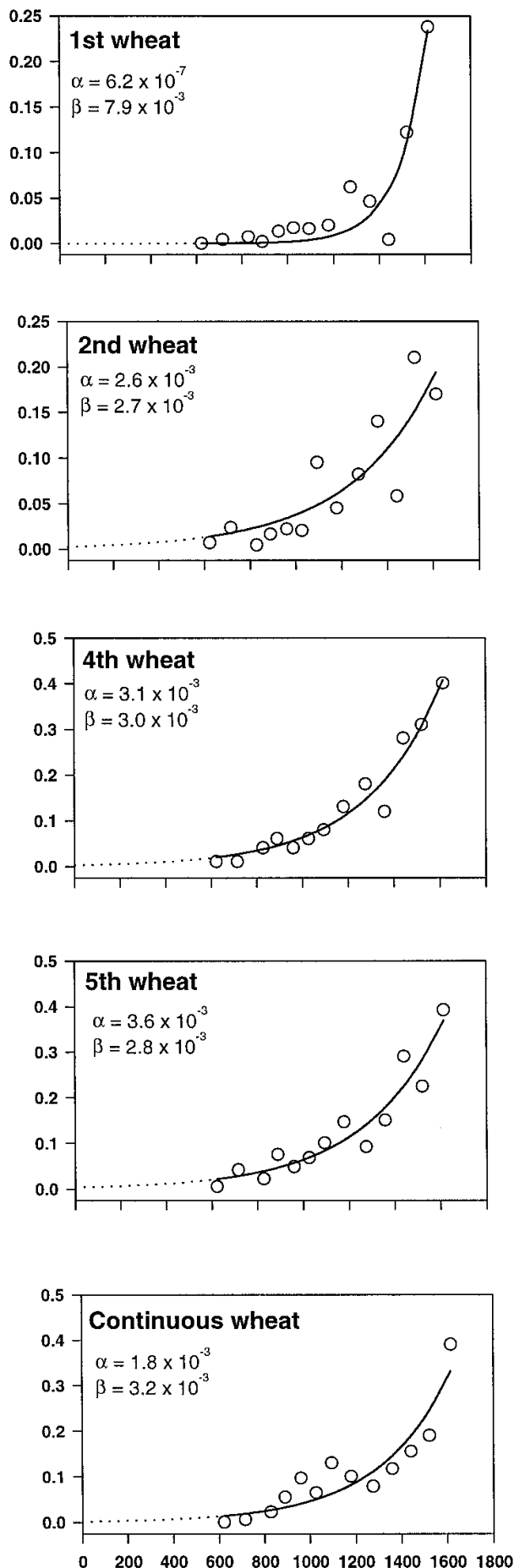

Time (Day degrees $>3{ }^{\circ} \mathrm{C}$ )

Fig. 4. Plots describing disease progress on A, seminal and $\mathbf{B}$, adventitious roots of wheat plants growing in naturally infected field plots at Rothamsted Experimental Station, England. The model for primary and secondary infection was fitted to the data for seminal roots, with the rate of inoculum decay $\left(r_{d}\right)$ fixed across cropping frequency. The density of seminal roots was assumed to be constant over the course of the experiment. Trends in disease progress for adventitious roots are described by simple exponential curves: $i=\beta \exp (\alpha t)$. Characteristic features of the curves include relatively high initial levels of take-all on seminal roots compared with the absence of disease on adventitious roots. Dashed lines indicate extrapolated curves that describe the primary infection of seminal roots consistent with controlled environment data. 
as temperature. In this paper, we used controlled environments to minimize periodic and stochastic changes in driving variables to understand the underlying population dynamics. The controlled environment experiments also allowed for multiple observations over time, something which is prohibitively expensive to do in the field. We showed that the density of initial inoculum can have a profound effect on the nature and shape of disease progress curves for take-all (Fig. 2). Reducing the density of initial inoculum switched the epidemic curve from a comparatively simple monomolecular approach toward an upper asymptote (Fig. 2A) to a stepped curve with an early monomolecular rise to a temporary plateau and thereafter a sigmoidal rise toward an asymptote (Fig. 2A). The resulting curves were disassembled to separate infection on seminal and adventitious roots and cautiously used to interpret disease progress curves in continuous wheat crops in the field. Field data originally were collected for an independent experiment and did not contain observations for the critical period of primary infection during the autumn, but careful extrapolation allowed us to summarize the characteristic shapes of the curves for the field data from sowing to mid-summer. We suggest that the shapes of the resulting curves (Fig. 4) are consistent with deconstructed curves for the intensively monitored controlled environment experiment in which the phases of secondary and primary infection are markedly affected by root and inoculum dynamics.

The change in curve shape at the lower inoculum density was consistent with a switch from an early phase driven by primary infection to a later phase controlled by secondary infection as the fungus spreads from infected to newly available susceptible roots. The results were compatible with predictions based on the epidemic model used by Gilligan and Kleczkowski (14), which synthesizes the dynamics of inoculum, host growth, and primary and secondary infection. Failure to include host and inoculum dynamics concealed the dynamic features of the epidemic, yielding curves that fit the data badly (compare Fig. 2A and D). Careful inspection of the residuals for the full model incorporating host and inoculum dynamics shows that the model fails to capture some of the detail of the plateau at low inoculum density (Fig. 2A and C). The fitted curve underestimated the duration of the initial plateau, which may reflect the absence of an interaction between the production of new susceptible roots and the level of infection on the seminal roots in the model. Underestimation of the duration of the plateau also may be associated with a prolonged latent period that is not explicitly treated in the model. Separation of these hypotheses requires further experimental testing.

Figure 5 is a schematic summary of take-all disease and inoculum dynamics. The deceleration of the primary phase of the epidemic was caused by the decay in infectivity of particulate inoculum and saturation of relatively low densities of susceptible roots, which are primarily restricted to the seminal root system, with its upper limit of only five, occasionally up to seven, roots per plant. Rapid decay of particulate inoculum of $G$. graminis var. tritici under field conditions has been well documented $(19,25)$ and is attributed to the organism's poor competitive saprophytic ability (8). Scott (24) also reported exponential decay in the dynamics of $G$. graminis var. tritici, although it is possible that under some circumstances, there may be a delay before decay, leading to a sigmoidal pattern of loss. Further independent support for the dominance of primary infection on seminal root systems was provided by the correspondence between the asymptotic level of primary infection estimated by the static probability model (Fig. 3A) and the plateau estimated by the dynamic model. Subsequent acceleration in the disease progress curve was initiated by secondary infection from roots previously infected by primary infection and corresponded with the increased availability of susceptible tissue as adventitious roots began to grow.

It is probable that delays in epidemics frequently observed in the field have been attributed solely to the onset of colder weather. Using controlled environments free of environmental forcing vari-

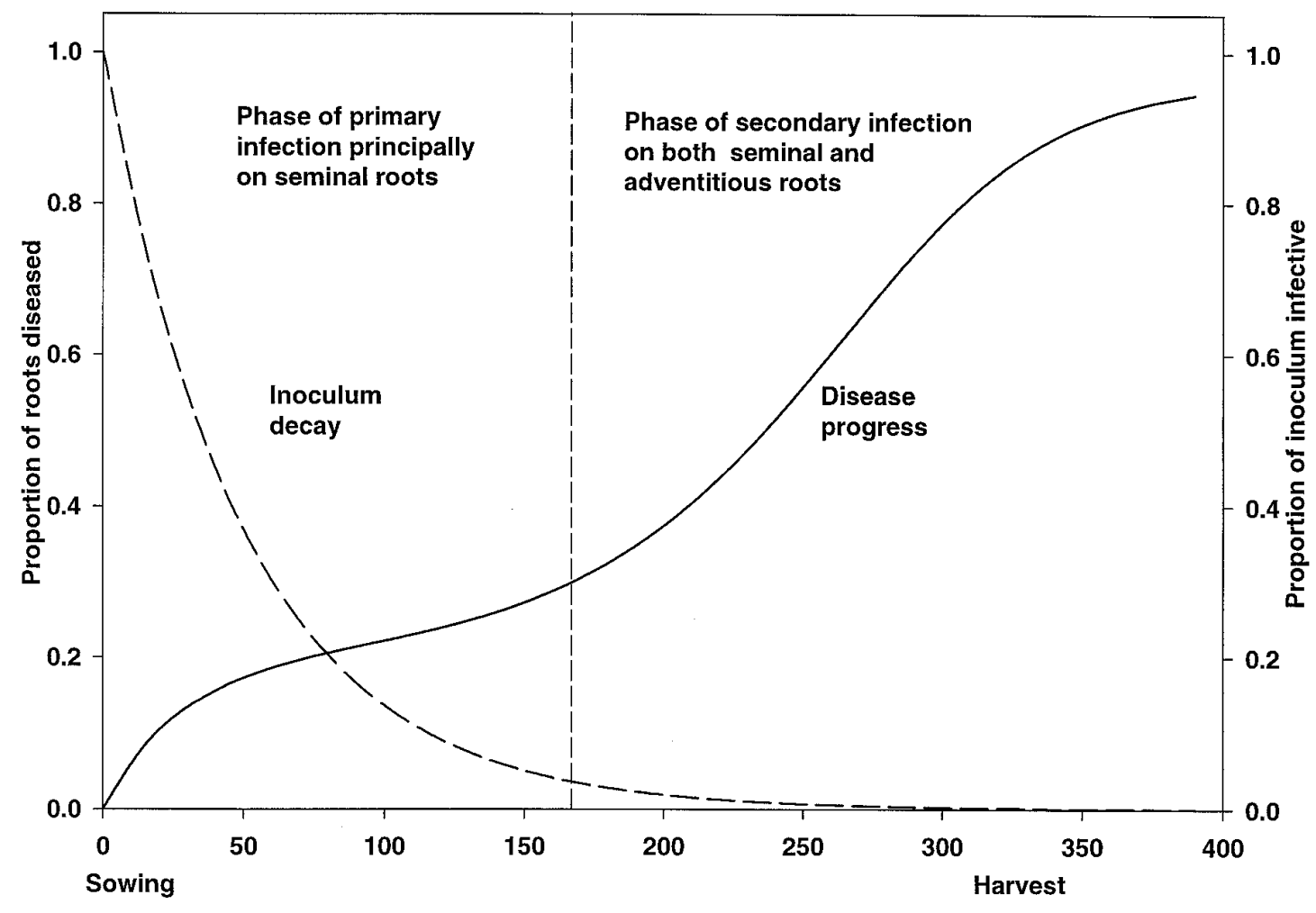

Time (Days after sowing)

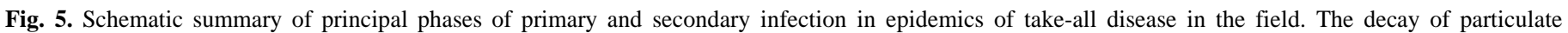
inoculum for primary infection derived from the previous susceptible crop is shown (dashed line). 
ables, we have shown that the plateau reflects the inherent nonlinear dynamics of initial inoculum, disease, and root growth. A period of colder weather would accentuate the effect. The description of disease progress with two plateaus is not restricted to infection of wheat by G. graminis var. tritici. Amorim et al. (1) and Hau et al. (18) reported similar patterns for Ustilago scitaminea on sugarcane, and Rupe and Gbur (23) reported similar patterns for Fusarium solani on soybean. Hau et al. (18) analyzed the double sigmoidal patterns of the data for Amorim et al. (1), using an analytical expression for the sum of two simple growth functions, but the current study is the first example involving interpretation of disease progress curves in relation to primary and secondary infection based on empirical analysis of the underlying biological components involved in host growth and inoculum dynamics.

The model we used to summarize the dynamics of the take-all epidemic is relatively simple, although it encompasses primary and secondary infection, host growth, and inoculum dynamics. In particular, the model relies on simple mass action terms for transmission of infection, implying complete mixing between inoculum and susceptible roots and between infected and susceptible roots, which is likely to apply to primary infection if we assume that inoculum is well dispersed by cultivation between crops. It is unlikely to apply to secondary infection, in which there is clustering of infected roots and spatial correlations of roots. We have shown previously how incomplete mixing can be incorporated into models for infection of Sclerotinia minor by Sporidesmium sclerotivorum $(16,17)$ and Raphanus sativus by Rhizoctonia solani (20) by incorporating powers in the mixing terms for infected and susceptible roots. The data, however, for G. graminis var. tritici did not merit this additional complication, although it is probable that incomplete mixing may be important in contributing to local heterogeneity in disease development in the field. Further elaborations are clearly possible in separating host growth into seminal and adventitious roots and incorporating a host response to infection (13), but the elaborations await further experimental evidence.

Separation of the epidemic into components for primary and secondary infection recently has been used to analyze the dynamics of Polymyxa betae, the beet necrotic yellow vein virus vector on sugar beet to identify critical phases for management of the disease $(26,27)$. Separation of the components will assist in the deployment of biological and chemical control of G. graminis var. tritici, because together with the dynamic model given in equation 1 , it enables analysis of the effects of transient control on subsequent disease development. Bull et al. (6) observed the effects of antagonistic Pseudomonas spp. on suppression of primary infection of G. graminis var. tritici as a reduction in lesion growth. This, in turn, affects the probability of secondary infection. Formal analysis of effects on epidemiological dynamics is still in its infancy yet greatly affects not only the prediction of the success of biological control but also helps to identify reasons for failure other than the inherent variability of biological control. We have shown previously for Rhizoctonia solani and Trichoderma viride that small changes in initial conditions can have profound effects on the dynamics of epidemics $(2,20,21)$ and may account for variability between epidemics. Work is underway to extend these analyses to the G. graminis var. tritici-Pseudomonas systems.

\section{ACKNOWLEDGMENTS}

This work was funded by a grant from the Biotechnology and Biological Sciences Research Council. We thank A. R. Werker and D. Hornby for permission to quote results from our previous joint study.

\section{LITERATURE CITED}

1. Amorim, L., Filho, A. B., and Hau, B. 1993. Analysis of progress curves of sugarcane smut on different cultivars using functions of double sigmoid pattern. Phytopathology 83:933-936.

2. Bailey, D. J., and Gilligan, C. A. 1997. Biological control of pathozone behaviour and disease dynamics of Rhizoctonia solani by Trichoderma viride. New Phytol. 136:359-367.

3. Brassett, P. R., and Gilligan, C. A. 1988. A model for primary and secondary infection in botanical epidemics. Z. Pflanzenkr. Pflanzenschutz 95: 352-360.

4. Brassett, P. R., and Gilligan, C. A. 1989. Fitting of simple models for field disease progress for the take-all fungus. Plant Pathol. 38:397407.

5. Briggle, L. W., and Curtis, B. C. 1987. Wheat worldwide. Pages 1-32 in: Wheat and Wheat Improvement. E. G. Heyne, ed. American Society of Agronomy and Crop Sciences of America, Madison, WI.

6. Bull, C. T., Weller, D. M., and Thomashow, L. S. 1991. Relationship between root colonization and suppression of Gaeumannomyces graminis var. tritici by Pseudomonas fluorescens strain 2-79. Phytopathology 81:954-959.

7. Colbach, N., Lucas, P., and Meynard, J. M. 1997. Influence of crop management on take-all development and disease cycles on winter wheat. Phytopathology 87:26-32.

8. Garrett, S. D. 1970. Pathogenic Root Infecting Fungi. Cambridge University Press, Cambridge.

9. Gilligan, C. A. 1985. Probability models for host infection by soilborne fungi. Phytopathology 75:61-67.

10. Gilligan, C. A. 1990. Mathematical models of infection. Pages 207232 in: The Rhizosphere. J. Lynch, ed. John Wiley \& Sons, Chichester, England.

11. Gilligan, C. A., and Brassett, P. R. 1990. Modelling and estimation of relative potential for infection of winter wheat by inoculum of Gaeumannomyces graminis derived from propagules and infected plants. J. Phytopathol. 129:58-68.

12. Gilligan, C. A., Brassett, P. R., and Campbell, A. 1994. Computer simulation of early infection of cereal roots by the take-all fungus: A detailed stochastic, mechanistic simulator. New Phytol. 128:515-527.

13. Gilligan, C. A., Gubbins, S., and Simons, S. A. 1997. Analysis and fitting of an SIR model with host response to infection load for a plant disease. Philos. Trans. R. Soc. Lond. Ser. B 352:353-364.

14. Gilligan, C. A., and Kleczkowski, A. 1997. Population dynamics of botanical epidemics involving primary and secondary infection. Philos. Trans. R. Soc. Lond. Ser. B 352:591-608.

15. Gilligan, C. A., and Simons, S. A. 1987. Inoculum efficiency and pathozone width for two host-parasite systems. New Phytol. 107:549-566.

16. Gubbins, S., and Gilligan, C. A. 1997. A test of heterogeneous mixing as a mechanism for ecological persistence in disturbed environments. Proc. R. Soc. Lond. Ser. B 264:227-232.

17. Gubbins, S., and Gilligan, C. A. 1997. Biological control in a disturbed environment. Philos. Trans. R. Soc. Lond. Ser. B 352:1935-1949.

18. Hau, B., Amorim, L., and Filho, A. B. 1993. Mathematical functions to describe disease progress curves of double sigmoid pattern. Phytopathology 83:928-932.

19. Hornby, D. 1981. Inoculum. Pages 271-294 in: Biology and Control of Take-all. M. J. C. Asher and P. J. Shipton, eds. Academic Press, New York.

20. Kleczkowski, A., Bailey, D. J., and Gilligan, C. A. 1996. Dynamically generated variability in a plant pathogen system with biological control. Proc. R. Soc. Lond. Ser. B. 263:777-783.

21. Kleczkowski, A., Gilligan, C. A., and Bailey, D. J. 1997. Scaling and spatial dynamics in plant pathogen systems: From individuals to populations. Proc. R. Soc. Lond. Ser. B. 264:979-984.

22. Ross, G. J. S. 1990. Nonlinear Estimation. Springer-Verlag, Heidelberg, Germany.

23. Rupe, J. C., and Gbur, E. E., Jr. 1995. Effect of plant age, maturity group, and environment on disease progress of sudden death syndrome of soybean. Plant Dis. 79:139-143.

24. Scott, P. R. 1969. Effects of nitrogen and glucose on saprophytic survival of Ophiobolus graminis in buried straw. Ann. Appl. Biol. 63:27-36.

25. Shipton, P. J. 1981. Saprophytic survival between susceptible crops. Pages 295-316 in: Biology and Control of Take-all. M. J. C. Asher and P. J. Shipton, eds. Academic Press, New York.

26. Truscott, J. E., Webb, C. R., and Gilligan, C. A. 1998. Asymptotic analysis of an epidemic model with primary and secondary infection. Bull. Math. Biol. 59:1101-1123.

27. Webb, C. R., Gilligan, C. A., and Asher, M. J. C. 1999. A model for the build-up of Polymyxa betae, the vector of rhizomania. Phytopathology 89:30-38.

28. Werker, A. R., Gilligan, C. A., and Hornby, D. 1991. Analysis of shapes of disease progress curves for take-all in consecutive winter wheat crops Plant Pathol. 40:8-25. 